\title{
Electron and hole spectra in quantum wire with two quantum dots in the electric field
}

\author{
O.Makhanets, A.Gryschyk, M.Dovganiuk \\ Fedkovych Chernivtsi National University, 2, Kotsyubinskoho Str., Chernivtsi, 58012, Ukraine*
}

Received September 4, 2006

\begin{abstract}
The energy spectrum of electron and hole is investigated in a complicated nanoheterosystem consisting of two cylindrical semiconductor quantum dots placed into semiconductor quantum wire. Quantum dots are separated by barrier-layer, which is under the action of a constant electric field. The dependencies of electron and hole energies on geometric parameters of quantum dots and electric field intensity are analysed.
\end{abstract}

Key words: quantum wire, quantum dot, energy spectrum

PACS: 73.21.Fg, 73.21. $\mathrm{Hb}, 73.21 . \mathrm{La}$

\section{Introduction}

Artificial atoms or quantum dots (QDs) constructed from semiconductors are expected to provide the basis for future generations of device technologies such as threshholdless lasers and ultradense memories. When several quantum dots are connected to each other, they provide remarkable phenomena due to the interplay of electron correlations, interference effects, etc, which depend on how the dots are arranged: e.g. double quantum dots coupled in series or parallel [1-5].

In this context double quantum dots are of particular interest from two different viewpoints such as implementation of quantum bits (qubit) [6,7] and a model system for molecular binding under controlled conditions $[8,9]$.

At present, of particular interest are the double quantum dots embedded into quantum nanowires. Such systems have already been created and are being intensively investigated experimentally [10,11].

The theory of spectra and wave functions of quasiparticles (electrons, holes, excitons) in quantum dots embedded into the quantum wires is only at the start of development [12]. Such combined nanoheterosystems are rather complicated for mathematical description due to the complicated fitting conditions for the wave functions of quasiparticles.

In this paper we are going to study the stationary energy states of electron and hole in a nanoheterosystem consisting of two semiconductor quantum dots (of different size) embedded into the cylindrical quantum wire. The thin barrier-layer separating quantum dots are under the action of a constant electric field of a fixed intensity $(\vec{F})$, directed along the axial axis

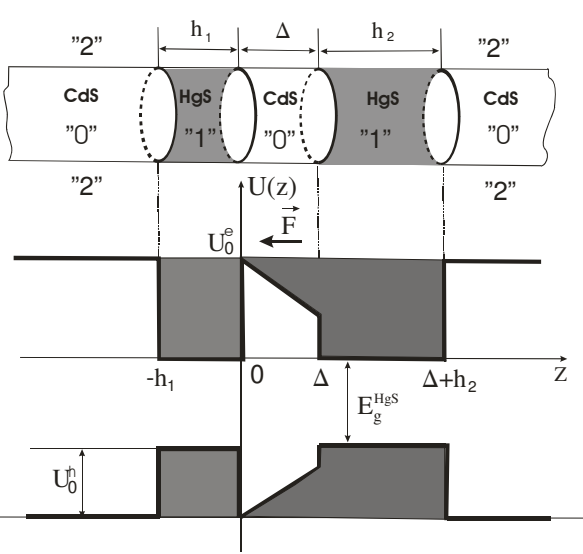

Figure 1. Geometrical scheme of a nanosystem and the dependence of electron and hole potential energy on $z$ variable. of nanohetrosystem (figure 1).

*E-mail: theorphys@chnu.cv.ua 


\section{The theory of electron (hole) energy spectrum in cylindrical quantum wire with two quantum dots inside}

The complicated semiconductor cylindrical quantum wire (" 0 "), containing two quantum dots of the same material (" 1 "), separated by a thin layer of another material (" 0 ") is under research. The radius of QW $\left(\rho_{0}\right)$, the height of QDs $\left(h_{1}\right.$ and $\left.h_{2}\right)$, respectively and thickness of the layer separating QDs $(\Delta)$ are assumed to be fixed (figure 1 ).

A complicated quantum wire is placed into the external medium forming the infinite potential barriers for the electron (hole). The electron (hole) effective masses are different in different parts of a nanosystem:

$$
\mu^{\mathrm{eh}}(z)= \begin{cases}\mu_{0}^{\mathrm{eh}}, & \text { medium "0", } \\ \mu_{1}^{\mathrm{eh}}, & \text { medium " } 1 " .\end{cases}
$$

It is also assumed that the lattice constants $a_{0}$ and $a_{1}$ of the media " 0 " and " 1 " are to be close in magnitude. For example, the computer calculations are performed for the nanosystem created at the base of $\beta-\mathrm{HgS}$ and $\beta-\mathrm{CdS}$ crystals where the lattice constants are so close that $\left(a_{1}-a_{0}\right) / a_{0} \leqslant 1 \%$. Since the interfaces between different parts of nanosystem are rather straight, it is possible to use the approximation of rectangular potential energies for the electron and hole.

Taking this into account as well as the fact that the constant electric field with intensity of $\vec{F}$ is applied to the barrier-layer ("0") like it is shown in figure 1, the potential energy can be written in the form

$$
\begin{aligned}
& U^{\mathrm{e}}(\rho, \varphi, z)=\left\{\begin{array}{ll}
U_{0}^{\mathrm{e}}, & z<-h_{1} \\
0, & -h_{1} \leqslant z \leqslant 0 \\
U_{0}^{\mathrm{e}}-e F z, & 0 \leqslant z \leqslant \Delta,
\end{array} \quad \text { and } \quad \Delta \leqslant \Delta+h_{2},\right. \\
& U^{\mathrm{h}}(\rho, \varphi, z)=\left\{\begin{array}{l}
-E_{g}^{\mathrm{HgS}}-U_{0}^{\mathrm{h}}, \quad z<-h_{1} \quad \text { and } z>\Delta+h_{2}, \\
-E_{g}^{\mathrm{HgS}}, \quad-h_{1} \leqslant z \leqslant 0 \quad \text { and } \Delta \leqslant z \leqslant \Delta+h_{2}, \\
-E_{g}^{\mathrm{HgS}}-U_{0}^{\mathrm{h}}+e F z, \quad 0 \leqslant z \leqslant \Delta,
\end{array}\right.
\end{aligned}
$$

where $e$ is the electron charge, $U_{0}^{\mathrm{eh}}=V_{0}^{\mathrm{eh}}-V_{1}^{\mathrm{eh}}, V_{0}^{\mathrm{e}}, V_{1}^{\mathrm{e}}\left(V_{0}^{\mathrm{h}}, V_{1}^{\mathrm{h}}\right)$ are the electron (hole) potential energies in the corresponding media " 0 " and " 1 ", taken respectively in vacuum.

The analytical calculation of electron and hole energy spectra and wave functions are equal, since the further formulas are presented for the electron omitting index $e$.

In order to investigate the electron quantum states, it is necessary to solve the Schrodinger equation.

$$
\widehat{H} \Psi(\vec{r})=E \Psi(\vec{r})
$$

with Hamiltonian

$$
\widehat{H}=-\frac{\hbar^{2}}{2} \vec{\nabla} \frac{1}{\mu(z)} \vec{\nabla}+U(\rho, \varphi, z)
$$

Taking into account the cylindrical symmetry it is convenient to write the wave function $\Psi(\tilde{\mathrm{r}})$ as in $[12]$

$$
\Psi_{n_{\rho} m}(\vec{r})=\left(-\pi \rho_{0}^{2} J_{m-1}\left(\chi_{n_{\rho} m}\right) J_{m+1}\left(\chi_{n_{\rho} m}\right)\right)^{-1 / 2} J_{m}\left(\frac{\chi_{n_{\rho} m}}{\rho_{0}} \rho\right) \mathrm{e}^{\mathrm{i} m \varphi} \varphi(z)
$$

where $m=0 ; \pm 1 ; \pm 2 ; \ldots$ is the magnetic quantum number, $J_{m}\left[\left(\chi_{n_{\rho} m}\right) / \rho_{0} \rho\right]$ is Bessel function of the whole order, $\chi_{n_{\rho} m}$ are roots of Bessel function $\left(n_{\rho}\right.$ is radial quantum number fixing the number of Bessel function root at the fixed $m$ ). 
Setting the wave function (6) in Schrodinger equation (4), the variables are separated and for $z$-th term of wave function there is obtained the equation

$$
\frac{\partial^{2}}{\partial z^{2}} \varphi(z)+\varphi(z)\left[\frac{2 \mu(z)}{\hbar^{2}}(E-U(\rho, \varphi, z))-\frac{\chi_{n_{\rho} m}^{2}}{\rho_{0}^{2}}\right]=0 .
$$

The solutions of equation (7) with notations

$$
k_{0}^{2}=\frac{2 \mu_{0}}{\hbar^{2}} E-\frac{\chi_{n_{\rho} m}^{2}}{\rho_{0}^{2}}, \quad k_{1}^{2}=\frac{2 \mu_{1}}{\hbar^{2}}\left(U_{0}-E\right)+\frac{\chi_{n_{\rho} m}^{2}}{\rho_{0}^{2}}
$$

for different parts of a nanosystem are as follows:

$$
\varphi(z)= \begin{cases}A^{+} \mathrm{e}^{k_{0} z}, & \mathrm{z}<-\mathrm{h}_{1}, \\ B^{+} \mathrm{e}^{\mathrm{i} k_{1} z}+B^{-} \mathrm{e}^{-\mathrm{i} k_{1} z}, & -h_{1} \leqslant z \leqslant 0, \\ C^{+} A i\left[-\xi\left(z+z_{B}\right)\right]+C^{-} B i\left[-\xi\left(z+z_{B}\right)\right], & 0 \leqslant z \leqslant \Delta, \\ D^{+} \mathrm{e}^{\mathrm{i} k_{1} z}+D^{-} \mathrm{e}^{-\mathrm{i} k_{1} z}, & \Delta \leqslant z \leqslant \Delta+h_{2}, \\ E^{-} \mathrm{e}^{-k_{0} z}, & z>\Delta+h_{2}\end{cases}
$$

here $A i(z), B i(z)$ are Airy functions of the first and second kind and

$$
\xi=\left(\frac{2 e F m}{\hbar^{2}}\right)^{1 / 3}, \quad z_{B}=\frac{E}{e F} .
$$

Using the conditions of a wave function and density of probability current continuity at the media interfaces of a nanosystem $\left(z=-h_{1}, z=0, z=\Delta, z=\Delta+h_{2}\right)$ and the condition of wave function normalization

$$
\int_{-\infty}^{\infty}|\varphi(z)|^{2} \mathrm{~d} z=1
$$

one can obtain analytical expressions for the coefficients $A^{+}, B^{ \pm}, C^{ \pm}, D^{ \pm}, E^{-}$(equation (8)) and a dispersion equation for defining the electron energies in a nanoheterosystem (the respective expressions are not presented because they are rather sophisticated). We should note that the electron (hole) wave function and its energy are characterized by three quantum numbers: $n_{\rho} m n_{z}$ $\left(\Psi_{n_{\rho} m n_{z}}^{\mathrm{eh}}(\vec{r}), E_{n_{\rho} m n_{z}}^{\mathrm{eh}}\right)$. The axial quantum number $\left(n_{z}\right)$ numerates the solutions of dispersion equation at the fixed $n_{\rho}$ and $m$ quantum numbers.

\section{Discussion of results}

The computer calculations of electron (hole) energy spectrum is performed for the nanoheterosystem created at the base of semiconductor crystals $\beta-\operatorname{HgS}$ ("1") and $\beta-\operatorname{CdS}$ ("0").

The results of calculating the electron and hole energy $\left(E_{n_{\rho} m n_{z}}^{\mathrm{eh}}\right)$ (without the electric field) as a function of the height of the second $\mathrm{QD}\left(h_{2}\right)$ at the fixed $n_{\rho}=1$, radius of $\mathrm{QW}: \rho_{0}=8 a_{\mathrm{HgS}}$, height of the other QD: $h_{1}=7 a_{\mathrm{HgS}}$ and width of the potential barrier: $\Delta=2 a_{\mathrm{CdS}}$ are presented in figure 2 .

Figure 2 proves that at $h_{2}=0$ there are three (two) electron (hole) energy levels in a nanosystem, coinciding (as it should be) with the levels arising in QD (HgS) with the height $h_{1}$ embedded into QW (CdS). The increase of $h_{2}$ height causes the appearance of new energy levels becoming smaller and creating anticrossings. They are caused by the splitting of the levels, having the origin of both potential wells, accounting for their interaction through the potential barrier with finite height and width. 

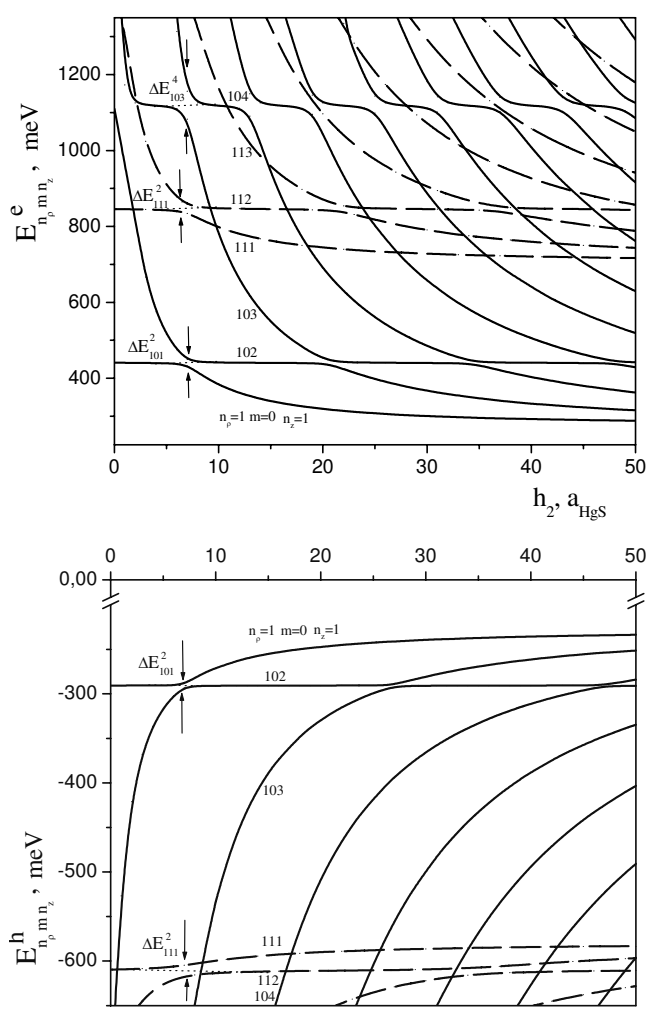

Figure 2. Dependences of electron and hole energies on the height of second QD $\left(h_{2}\right)$ at $n_{\rho}=1$, $\rho_{0}=8 a_{\mathrm{HgS}}, h_{1}=7 a_{\mathrm{HgS}}, \Delta=2 a_{\mathrm{CdS}}, F=0$.

The number of energy levels is determined by the volume of quantum wells of the system. It should be mentioned that at the equal geometric parameters, the number of electron energy states is much bigger than of the hole ones. This is obviously caused by the fact that the depth of a potential well for the electron $\left(U_{0}^{\mathrm{e}}\right)$ is bigger than twice the respective magnitude for the hole $\left(U_{0}^{\mathrm{h}}\right)$.

From the general analysis of the dispersion equation it is clear that all electron and hole energy levels (except the ground level) are twice degenerated in respect to the magnetic quantum number $m$. Besides, as one can see in figure 2 there is a casual degeneration of energy levels because the levels with different $n_{z}$ and $m$ are crossing at the variation of $h_{2}$.

The application of the electric field $\vec{F}$ does not qualitatively change the behavior of electron (hole) energy spectrum but causes the shift of all energy levels into the region of smaller energies, to the small shift of all anticrossings into the region of smaller magnitudes of $h_{2}$ and to the varying of the splitting of energy levels with equal symmetry $\Delta E_{n_{\rho}}^{(e, h) n_{z}^{\prime}}$. It is seen in figure $3 \mathrm{a}$,b, where there are presented the dependences of electron (a) and hole (b) energies on the height of the quantum dot $h_{2}$ (in the range of $2-9 a_{\mathrm{HgS}}$ ) at the fixed $h_{1}=7 a_{\mathrm{HgS}}, \Delta=2 a_{\mathrm{CdS}}, \rho_{0}=8 a_{\mathrm{HgS}}$ when there is no electric field $F=0$ (solid curves) and for the electric field with the intensity $F=580 \mathrm{MV} / \mathrm{m}$ (dash curves). In the figures one can see that the increase of intensity $\vec{F}$ causes the increase of the splitting $\Delta E_{n_{\rho} m n_{z}}^{(e, h)}$. The latter is bigger and increases faster with an increase of the anticrossings in the energy scale of quantum well. It should be mentioned that the increase of the splitting for the higher energy levels is capable of reaching $100 \mathrm{meV}$. Such a behavior of energy spectrum is clear from physical viewpoint. Really, the increase of the electric field intensity causes the change of potential barrier profile in such a way that, on the one hand, its effective thickness becomes smaller, i.e., its transparency increases, obviously increasing the interaction between quantum wells and, consequently, bringing to the increase of the splitting of the respective energy levels. On the other hand, the increase of transparency effectively increases the quantum well volume (the toned region 

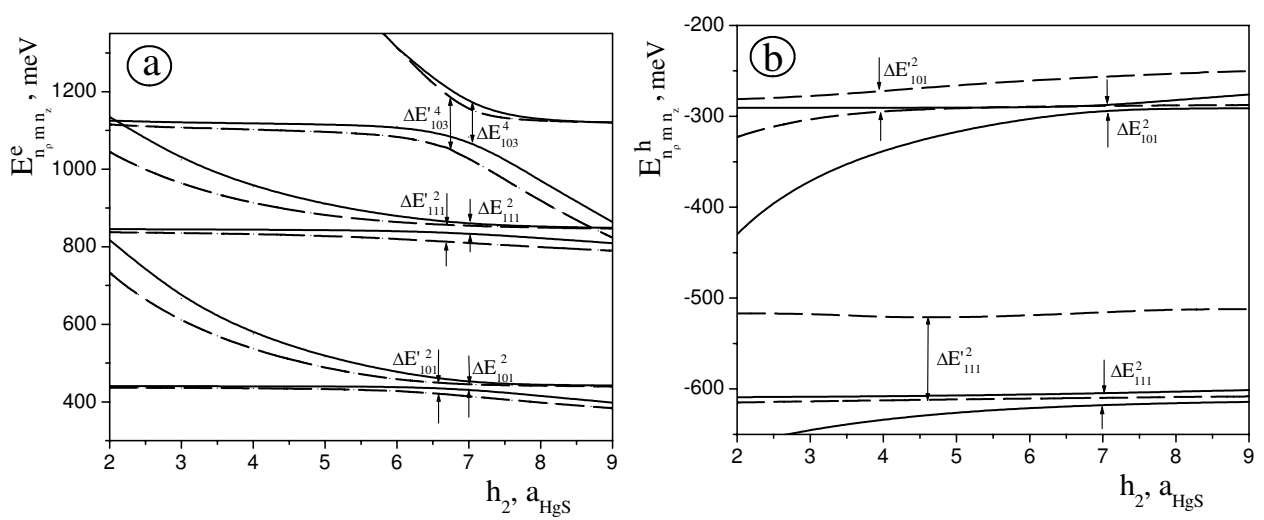

Figure 3. Dependences of electron (a) and hole (b) energies on the height of quantum dot $h_{2}$ at the fixed $h_{1}=7 a_{\mathrm{HgS}}, \Delta=2 a_{\mathrm{CdS}}, \rho_{0}=8 a_{\mathrm{HgS}}$ when there is no electric field: $F=0$ (solid curves) and for the electric field with intensity $F=580 \mathrm{MV} / \mathrm{m}$ (dash curves).

$0 \leqslant z \leqslant \Delta+h_{2}$ in figure 1). On the one hand, it decreases the electron and hole energy and, on the other hand, causes the anticrossing arising at the smaller values of the second quantum dot $\left(h_{2}\right)$.

The final remark is that even an inessential shift of electron and hole energy levels into the region of smaller energies at the increase of the electric field intensity $\vec{F}$ can essentially effect the binding and exciting energy of exciton in the system under research. The detailed calculation and analysis of exciton spectra are to be performed in future.

\section{References}

1. Aono T., Eto M., Kawamura K. J. Phys. Soc. Jpn., 1998, 67, 1860.

2. Georges A., Meir Y. Phys. Rev. Lett. 1999, 82, 3508.

3. Aguado R., Langreth D.C. Phys. Rev. Lett., 2000, 85, 1946.

4. Izumida W., Sakai O. Phys. Rev. B, 2000, 62, 10260.

5. Aono T., Eto M. Phys. Rev. B, 2001, 63, 125327.

6. Hu X., Sarma S.D. Phys. Rev. A, 2000, 61, 062301.

7. Burkard G., Seelig G., Loss D. Phys. Rev. B, 2000, 62, 2581.

8. Partoens B., Peeters F. Europhys. Lett., 2001, 56, 86.

9. Amaha S., Austing D.G., Tokura Y., Muraki K., Ono K., Tarucha S. Solid State Commun., 2001, 119, 183.

10. Bjork M.T., Thelander C., Hansen A.E., Jensen L.E., Larsson M.W., Wallenberg L.R., Samuelson L. Nano Lett., 2004, 4, 1621.

11. Fasth C., Fuhrer A., Bjork M.T., Samuelson L. Nano Lett., 2005, 5, 1487.

12. Tkach N.V., Makhanets A.M. Fiz. Tv. Tela, 2005, 47, 550 (in Russian). 


\section{Спектри електрона та дірки у квантовому дроті з двома квантовими точками в електричному полі}

\section{О.М.Маханець, А.М.Грищук, М.М.Довганюк}

Чернівецький національний університет ім. Юрія Федьковича, вул. Коцюбинського 2, 58012, Чернівці

Отримано 4 вересня 2006 р.

Досліджено енергетичний спектр електрона і дірки в складній наногетеросистемі, що складається 3 двох циліндричних напівпровідникових квантових точок, розташованих у напівпровідниковому квантовому дроті. Квантові точки розділені шаром-бар'єром, до якого прикладене постійне електричне поле. Проаналізовано залежності енергій електрона і дірки від геометричних розмірів квантових точок і величини напруженості електричного поля.

Ключові слова: квантовий дріт, квантова точка, енергетичний спектр

PACS: 73.21.Fg, 73.21. Hb, 73.21.La 